\title{
Profesionalismo en la enseñanza del idioma inglés (ELT) \\ Resultados preliminares de un estudio etnográfico
}

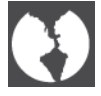

RESUMEN
Frank Farmer*

María Elena Llaven Nucamendi** Amalfi Novelo Granados

En el estudio del profesionalismo existen muchas opiniones sobre la naturaleza del fenómeno, pero pocas explicaciones de cómo funciona y con qué propósito. En este estudio se toma como base la explicación coherente del profesionalismo que ofrecen Dingwall y Fenn.

El análisis del modelo de profesionalismo de estos investigadores permite una categorización de los trabajos que realizan profesiones específicas, dentro de un marco que se deriva de las acciones necesarias en cualquier profesión para cumplir con el modelo. Para el estudio de English Language Teaching (ELT), donde faltan normas de trabajo y un colegio profesional, ofrece una oportunidad para medir en términos cuantificables qué tan profesionales son sus acciones, y sacar comparaciones con profesiones establecidas.

El presente estudio toma como puntos de referencia el profesionalismo de médicos y arquitectos en el Reino Unido. Ambas profesiones cuentan con la estructura de una profesión muy desarrollada, con su colegio de profesionistas, el monopolio legal de su campo de conocimiento, procedimientos de interna, y un ámbito legal donde las demandas por negligencia e incompetencia proceden con transparencia.

Los resultados del presente estudio muestran que a pesar de la ausencia de los órganos profesionales y un sistema legal

\footnotetext{
*Universidad de Quintana Roo / frank@uqroo.mx

** Universidad de Quintana Roo / marllave@uqroo.mx
} 
adecuado, la práctica de elT en México no está tan lejos del modelo propuesto. Algunos de los resultados obtenidos no pueden ser analizados con más detalle para proponer explicaciones adecuadas, además, las muestras de arquitectos y médicos no son suficientes para sacar estadísticas confiables. Este trabajo pertenece a una segunda etapa de la investigación.

Palabras Disciplina, elt, profesionalismo, responsabilidad.

CLAVE

\section{Introducción}

En el estudio del profesionalismo existen varias opiniones, positivas y negativas. Las negativas critican el monopolio del trabajo que supuestamente tienen los profesionistas (Illich et al., 1977; Larson, 1977), y ponen en duda la validez de sus habilidades y conocimientos (Goode, 1969; Illich et al., 1977). Los sustentantes del profesionalismo de las opiniones positivas responden a estos críticos planteando que I) el monopolio del trabajo está sujeto a condiciones impuestas por la sociedad y éstas son necesarias para proteger al público de charlatanes, y 2) no hay otra forma en la que el público pueda tener acceso a los servicios de verdaderos expertos (Abbott, 1988; Dingwall y Fenn, 1987; Freidson, 200I). Las opiniones positivas señalan que el profesionalismo lo constituyen las acciones tomadas por los verdaderos expertos para la solución de problemas, y estas acciones están controladas por el Estado para la protección del público. De acuerdo con este punto de vista, el profesionalismo no consiste en la formación de cuerpos de profesionistas que restrinjan la selección del perfil profesional del profesionista que el público quiera emplear, ni tampoco consiste en escribir códigos de ética que presenten dificultades para su seguimiento.

Por lo tanto, para estudiar el nivel del profesionalismo de algún grupo de trabajadores expertos, sería conveniente realizar un análisis de sus acciones, los conocimientos que guían las acciones, y la manera en que adquieren los conocimientos. Para establecer un nivel de referencia es necesario aplicar el mismo instrumento que se utiliza en profesiones que cuentan con excelencia reconocida. 


\section{Diseño del estudio}

Dingwall y Fenn (I987) analizaron la naturaleza del profesionalismo en su contexto económico y legal y presentaron un esquema coherente de la legitimidad de la formación de colegios de profesionistas para proteger las áreas de trabajo especializadas. Dingwall y Fenn basaron su explicación en los principios del mercado libre, donde por consenso general no deben existir monopolios. El problema es como tratar la práctica del conocimiento y competencia especializada donde el derecho de monopolio de trabajo es necesitado para proteger al público de practicantes' incompetentes, contradiciendo los principios de un mercado libre. Estos autores argumentan que un órgano colegiado de profesionistas es necesario para el control tanto del ingreso como del desarrollo profesional continuo. Sin embargo, los colegios de profesionistas son llamados no solamente en la defensa de los derechos de sus miembros sino también en la defensa de los derechos de los clientes en casos específicos de incompetencia profesional, porque solamente el colegio de profesionistas es competente para juzgar la calidad del trabajo profesional. Los autores identifican dos principales problemas con esta situación: el primer problema es la asimetría informativa de la relación entre el profesional y su cliente, donde el cliente no tiene manera de conocer si el servicio dado podría ser mejor o si un resultado indeseable podría ser evitado; y el segundo problema es la transparencia de la regulación de los profesionistas por los profesionistas mismos ¿Quién regula al regulador?

Las profesiones no son ocupaciones de servicios ordinarios, argumentan los autores. Su posición especial requiere que ellos sean responsables de las decisiones difíciles de sus clientes, donde "el juicio del profesional estabiliza lo imprevisible dentro de una base suficientemente confiable para la acción humana" (Dingwall y Fenn, 1987: 6I). Su responsabilidad ante el Estado es ayudar, creando las condiciones de confianza requeridas para que exista una sociedad de mercado libre.

Las profesiones, entonces, son monopolios legítimos, y por lo tanto tienen que justificar sus prácticas a través del sistema legal. Los autores reconocen

' En este artículo el termino 'practicante' se refiere al que ejerce la profesión de alguna de las profesiones a las que se refiere el estudio, esto es, médico, arquitecto o profesor(a) de inglés. 
que la profesión legal de esta manera puede encontrarse fuera de la ley hasta cierto punto y esto presenta una problemática para su análisis. Pero para otras profesiones hay un trato implícito entre el Estado y la profesión, los términos de este trato están abiertos a negociación. El abuso del privilegio en una profesión puede ser castigado con el retiro de su monopolio. Los autores sugieren que la tensión causada por la inseguridad del monopolio, junto con litigación efectiva en casos de incompetencia y negligencia es al mismo tiempo la garantía de la responsabilidad del profesionista ante los clientes individuales y ante el Estado. Rechazan, por las mismas razones, la eliminación de monopolios de profesionistas así como el creciente control del Estado, puesto que estas medidas no son necesarias ni deseables en el mercado libre.

El punto de vista sobre el profesionalismo de Dingwall y Fenn resalta diferencias importantes entre las industrias de servicio y las profesiones. Los profesionistas son incapaces de decir de antemano con precisión qué servicios pueden necesitar los clientes de ellos, y por lo tanto, son incapaces de establecer las limitaciones de su deber al cliente y las condiciones que se apliquen al servicio. Resulta que llevar a cabo un ejercicio profesional implica cuando menos tres deberes ineludibles. Primero, los profesionistas deben ser expertos en el campo de los servicios que ofrecen. Segundo, deben tener conocimientos y habilidades para iniciar su servicio con un diagnostico de las necesidades del cliente. Tercero, tienen que prescribir la acción apropiada, ya sea por el profesionista mismo o por otro, a quien el profesionista considere el mejor preparado para lo que el cliente necesita. Lo anterior implica que los profesionistas deben mantener una bitácora de decisiones importantes, y además deben saber cómo proceder dentro de los límites de su competencia profesional en circunstancias difíciles y reales donde "el juicio del profesionista estabiliza lo impredecible dentro de una base lo suficientemente confiable para la acción humana".Y, finalmente, los profesionistas necesitan mantenerse al corriente, no solamente de los avances en su área del conocimiento, sino también de los casos legales que pueden afectar su responsabilidad. De esta manera se puede proponer que el trabajo de profesionistas cae dentro de las siguientes áreas:
A) Conocimiento del área especialista
B) Diagnosis
C) Prescripción 
D) Referencia a especialistas

E) Administración

F) Práctica general

G) Desarrollo profesional

\section{Recolección de datos}

Fueron elaborados cuestionarios acerca del trabajo de profesionistas, de acuerdo con las áreas de trabajo profesional identificadas. Estos cuestionarios se aplicaron a una muestra de 12 médicos y 10 arquitectos en el Reino Unido. Los participantes, médicos y arquitectos, fueron tomados como una referencia "benchmark" en este estudio, para medir el profesionalismo relativo de una muestra de 106 profesores de inglés como lengua extranjera en México. No se aplicaron técnicas estadísticas para el análisis de los resultados, por el tamaño de la muestra y por la composición del estudio. Por otro lado, el estudio no busca hacer generalizaciones fuera de la muestra analizada. Los resultados son reportados en porcentajes expresados en números enteros.

Hipótesis I. Los profesionistas pueden concebir su trabajo dentro de la estructura sugerida.

Datos en apoyo a esta hipótesis pueden encontrarse en las respuestas de todo el cuestionario. Si los encuestados son capaces de reconocer algún aspecto de su trabajo en cada una de las siete secciones del cuestionario, la hipótesis sería confirmada. Los resultados de un análisis de respuestas por sección son dadas en la tabla $\mathrm{I}$.

De las tres profesiones estudiadas solamente Arquitectura da los resultados esperados, esto es, $100 \%$ en todas las categorías. Le sigue Medicina y luego ELT a mayor distancia del ideal.

Por otro lado, es posible para un practicante encontrar aspectos de su trabajo en cada una de las categorías, pero no necesariamente estar totalmente de acuerdo en que todas estas categorías realmente reflejan su trabajo. El que el estudio le imponga una estructura en la que tiene que categorizar su trabajo lo obliga a buscar la relación con los aspectos de su profesión. Esto se puede observar en la declaración especifica en la sección $F$ de cada cuestionario dirigida a este problema, pregunta P22 en ELT, PI7 en Medicina y 
TABLA I. Respuestas POR SECCIÓN DEL CUESTIONARIO.

\begin{tabular}{lccc}
\hline & ELT (\%) & Medicina (\%) & Arquitectura (\%) \\
\hline $\begin{array}{l}\text { A) Conocimiento } \\
\quad \text { del área especialista }\end{array}$ & 100 & 100 & 100 \\
B) Diagnosis & 99 & 100 & 100 \\
C) Prescripción & 98 & 83 & 100 \\
D) Referencia a especialistas & 80 & 92 & 100 \\
E) Administración & 82 & 100 & 100 \\
F) Práctica general & 100 & 100 & 100 \\
G) Desarrollo profesional & 100 & 100 & 100 \\
& & &
\end{tabular}

Arquitectura, que dice: "Mi trabajo es entregar un servicio profesional de diagnosis, prescripción, referencia a especialistas y mantener bitácoras”. Las respuestas a esta declaración son evaluadas de tal manera que $60 \%$ indicaría una posición no comprometida, mientras $100 \%$ indicaría acuerdo completo con todos los encuestados. Los participantes de ELT mostraron $60 \%$, lo que significa su alejamiento de la declaración. Médicos y arquitectos encuestados, por otro lado, sacaron porcentajes de $80 \%$ y $82 \%$ respectivamente, mostrando un firme acuerdo.

La diferencia sustancial entre ELT por un lado, y Medicina y Arquitectura por otro, puede reflejar una real divergencia en la manera en que estos dos grupos ven su trabajo. La hipótesis es sustentada por las respuestas dadas, pero sugiere que los practicantes de ELT no ven su trabajo en los términos profesionales usados en este estudio.

Hipótesis 2. Los profesionistas usualmente aprenden a hacer su trabajo en estudios de licenciatura en cualquier área del conocimiento, estudiando por su cuenta y experiencia práctica, pero no por estudiar posgrados.

Todos los encuestados en Medicina y Arquitectura afirmaron estar calificados para ejercer su profesión, pero la situación fue menos clara en ELT. Instituciones participantes en el proyecto aplicaron el cuestionario solamente al personal que consideraron calificado. No obstante, solamente $62 \%$ de los encuestados 
manifestaron estar calificados. En las tres profesiones relativamente pocos practicantes poseen un grado de posgrado en maestría (todos o la mayoría posee diplomados y/o estudios de licenciatura) e incluso pocos poseen doctorados. Esto refleja que en los requerimientos para admisión en los colegios de profesionistas el nivel de posgrado no es requerido. La tabla 2 compara los resultados de cada sección por profesión y global de cada una de las profesiones estudiadas.

La sección de Conocimiento del área especializada para Medicina y Arquitectura tiene dos preguntas acerca de la adquisición de conocimiento y tres preguntas en ELT. Los resultados de las respuestas fueron promediadas y se llegó a un solo porcentaje, que permitió hacer comparaciones. En esta sección, los encuestados sintieron la necesidad de extender las opciones dadas, quizá porque en la primera pregunta no es evidente si habrá más adelante la oportunidad de nombrar otras formas de aprendizaje.

En ELT, los encuestados señalaron que estudios de maestría fueron convenientes en todas las seis áreas de actividad profesional, aunque eso de ningún modo significa que aquellos estudios fueron lo más importantes en ninguna de las áreas. Los estudios doctorales ayudaron a médicos en todas las seis áreas, y los de maestría en dos. Arquitectos aplicaron conocimiento ganado en grados de maestría en sólo tres áreas de las seis, y ninguno posee doctorado. De nuevo, los posgrados no son los más frecuentemente citados como fuente de conocimiento. El orden relativo de importancia de las diferentes maneras de adquirir conocimiento, promediado en las seis áreas en cada profesión, está presentado en la tabla 6 , en la sección denominada promedio global. En todas las profesiones el valor de un posgrado viene en último lugar, confirmando la hipótesis en cuanto a la utilidad de un posgrado.

Los tres grupos de practicantes adquirieron conocimiento del área específica principalmente por estudiar por su cuenta diplomados y estudios de licenciatura, así como capacitación en servicio y experiencia, quizá reflejando la importancia de recibir educación antes de ejercer la profesión, así como capacitación en servicio. Hay diferencia entre ELT y las otras profesiones en la manera en que el conocimiento útil es adquirido, esto se muestra en otras secciones del cuestionario.

Los practicantes ELT aprenden más que los demás en diplomados y estudios de licenciatura, en lo que se refiere a las áreas de diagnosis, 
TABLA 2. UTILIDAD DE CALIFICACIONES ACADÉMICAS PARA ENTREGAR EL SERVICIO.

\begin{tabular}{|c|c|c|c|c|c|c|}
\hline Área de trabajo & ELT & $\%$ & Medicina & $\%$ & Arquitectura & $\%$ \\
\hline \multirow{8}{*}{$\begin{array}{l}\text { A) Conocimiento } \\
\text { del área } \\
\text { especialista }\end{array}$} & $\begin{array}{l}\text { Estudiar por } \\
\text { su cuenta }\end{array}$ & 53 & $\begin{array}{r}\text { Estudiar por } \\
\text { su cuenta }\end{array}$ & 46 & $\begin{array}{l}\text { Estudiar por } \\
\text { su cuenta }\end{array}$ & 50 \\
\hline & Diploma & 54 & Diploma & 8 & Diploma & 50 \\
\hline & Licenciatura & 36 & Licenciatura & 71 & Licenciatura & 65 \\
\hline & Maestría & 6 & Maestría & 13 & Maestría & 5 \\
\hline & Doctorado & I & Doctorado & 4 & Doctorado & 0 \\
\hline & $\begin{array}{l}\text { Capacitación } \\
\text { en servicio }\end{array}$ & 36 & $\begin{array}{r}\text { Capacitación } \\
\text { en servicio }\end{array}$ & 63 & $\begin{array}{l}\text { Capacitación } \\
\text { en servicio }\end{array}$ & 55 \\
\hline & Experiencia & 57 & Experiencia & 67 & Experiencia & 50 \\
\hline & Intuición & 16 & Intuición & 25 & Intuición & 5 \\
\hline \multirow[t]{8}{*}{ B) Diagnosis } & $\begin{array}{l}\text { Estudiar por } \\
\text { su cuenta }\end{array}$ & 42 & $\begin{array}{r}\text { Estudiar por } \\
\text { su cuenta }\end{array}$ & 50 & $\begin{array}{l}\text { Estudiar por } \\
\text { su cuenta }\end{array}$ & 50 \\
\hline & Diploma & 57 & Diploma & 17 & Diploma & 20 \\
\hline & Licenciatura & 32 & Licenciatura & 33 & Licenciatura & 0 \\
\hline & Maestría & 9 & Maestría & 8 & Maestría & 0 \\
\hline & Doctorado & 1 & Doctorado & 8 & Doctorado & 0 \\
\hline & $\begin{array}{r}\text { Capacitación } \\
\text { en servicio }\end{array}$ & 50 & $\begin{array}{r}\text { Capacitación } \\
\text { en servicio }\end{array}$ & 83 & $\begin{array}{r}\text { Capacitación } \\
\text { en servicio }\end{array}$ & 20 \\
\hline & Experiencia & 69 & Experiencia & 75 & Experiencia & 90 \\
\hline & Intuición & 27 & Intuición & 33 & Intuición & 40 \\
\hline \multirow[t]{8}{*}{ C) Prescripción } & $\begin{array}{l}\text { Estudiar por } \\
\text { su cuenta }\end{array}$ & 52 & $\begin{array}{l}\text { Estudiar por } \\
\text { su cuenta }\end{array}$ & 58 & $\begin{array}{l}\text { Estudiar por } \\
\text { su cuenta }\end{array}$ & 40 \\
\hline & Diploma & 58 & Diploma & 8 & Diploma & 20 \\
\hline & Licenciatura & 36 & Licenciatura & 33 & Licenciatura & 10 \\
\hline & Maestría & 11 & Maestría & 0 & Maestría & 10 \\
\hline & Doctorado & 1 & Doctorado & 8 & Doctorado & 0 \\
\hline & $\begin{array}{l}\text { Capacitación } \\
\text { en servicio }\end{array}$ & 47 & $\begin{array}{r}\text { Capacitación } \\
\text { en servicio }\end{array}$ & 58 & $\begin{array}{l}\text { Capacitación } \\
\text { en servicio }\end{array}$ & 30 \\
\hline & Experiencia & 68 & Experiencia & 58 & Experiencia & 90 \\
\hline & Intuición & 23 & Intuición & 17 & Intuición & 30 \\
\hline
\end{tabular}


TABLA 2. UTILIDAD DE CALIFICACIONES ACADÉMICAS (CONTINUACIÓN).

\begin{tabular}{|c|c|c|c|c|c|c|}
\hline Área de trabajo & ELT & $\%$ & Medicina & $\%$ & Arquitectura & $\%$ \\
\hline \multirow[t]{8}{*}{$\begin{array}{l}\text { D) Referencia a } \\
\text { especialistas }\end{array}$} & $\begin{array}{l}\text { Estudiar por } \\
\text { su cuenta }\end{array}$ & 42 & $\begin{array}{r}\text { Estudiar por } \\
\text { su cuenta }\end{array}$ & 50 & $\begin{array}{l}\text { Estudiar por } \\
\text { su cuenta }\end{array}$ & 20 \\
\hline & Diploma & 40 & Diploma & 17 & Diploma & 10 \\
\hline & Licenciatura & 30 & Licenciatura & 25 & Licenciatura & 10 \\
\hline & Maestría & 7 & Maestría & 0 & Maestría & 0 \\
\hline & Doctorado & 2 & Doctorado & 8 & Doctorado & 0 \\
\hline & $\begin{array}{r}\text { Capacitación } \\
\text { en servicio }\end{array}$ & 30 & $\begin{array}{r}\text { Capacitación } \\
\text { en servicio }\end{array}$ & 83 & $\begin{array}{r}\text { Capacitación } \\
\text { en servicio }\end{array}$ & 40 \\
\hline & Experiencia & 57 & Experiencia & 75 & Experiencia & 100 \\
\hline & Intuición & 17 & Intuición & 25 & Intuición & 20 \\
\hline \multirow[t]{8}{*}{$\begin{array}{l}\text { E) Adminis- } \\
\text { tración }\end{array}$} & $\begin{array}{l}\text { Estudiar por } \\
\text { su cuenta }\end{array}$ & 38 & $\begin{array}{r}\text { Estudiar por } \\
\text { su cuenta }\end{array}$ & 58 & $\begin{array}{l}\text { Estudiar por } \\
\text { su cuenta }\end{array}$ & 30 \\
\hline & Diploma & 33 & Diploma & 0 & Diploma & 10 \\
\hline & Licenciatura & 28 & Licenciatura & 33 & Licenciatura & 10 \\
\hline & Maestría & 8 & Maestría & 0 & Maestría & 0 \\
\hline & Doctorado & I & Doctorado & 8 & Doctorado & 0 \\
\hline & $\begin{array}{r}\text { Capacitación } \\
\text { en servicio }\end{array}$ & 30 & $\begin{array}{r}\text { Capacitación } \\
\text { en servicio }\end{array}$ & 92 & $\begin{array}{l}\text { Capacitación } \\
\text { en servicio }\end{array}$ & 40 \\
\hline & Experiencia & 59 & Experiencia & 75 & Experiencia & 90 \\
\hline & Intuición & 14 & Intuición & 33 & Intuición & 10 \\
\hline \multirow[t]{8}{*}{$\begin{array}{l}\text { F) Práctica } \\
\text { general }\end{array}$} & $\begin{array}{l}\text { Estudiar por } \\
\text { su cuenta }\end{array}$ & 44 & $\begin{array}{r}\text { Estudiar por } \\
\text { su cuenta }\end{array}$ & 58 & $\begin{array}{l}\text { Estudiar por } \\
\text { su cuenta }\end{array}$ & 20 \\
\hline & Diploma & 54 & Diploma & 17 & Diploma & 20 \\
\hline & Licenciatura & 35 & Licenciatura & 17 & Licenciatura & 10 \\
\hline & Maestría & 8 & Maestría & 0 & Maestría & 0 \\
\hline & Doctorado & I & Doctorado & 8 & Doctorado & 0 \\
\hline & $\begin{array}{r}\text { Capacitación } \\
\text { en servicio }\end{array}$ & $4 I$ & $\begin{array}{r}\text { Capacitación } \\
\text { en servicio }\end{array}$ & 92 & $\begin{array}{l}\text { Capacitación } \\
\text { en servicio }\end{array}$ & 30 \\
\hline & Experiencia & 63 & Experiencia & 75 & Experiencia & 100 \\
\hline & Intuición & 19 & Intuición & 33 & Intuición & 30 \\
\hline
\end{tabular}


TABLA 2. UtILIDAD DE CALIFICACIONES ACADÉMICAS PARA ENTREGAR EL SERVICIO.

(CONCLUYE)

\begin{tabular}{rrrrrrr}
\hline Área de trabajo & ELT & $\%$ & Medicina & $\%$ & Arquitectura & $\%$ \\
\hline Promedio global & $\begin{array}{r}\text { Estudiar por } \\
\text { su cuenta }\end{array}$ & 45 & $\begin{array}{r}\text { Estudiar por } \\
\text { su cuenta }\end{array}$ & 53 & $\begin{array}{r}\text { Estudiar por } \\
\text { su cuenta }\end{array}$ & 35 \\
Diploma & 49 & Diploma & II & Diploma & 22 \\
& Licenciatura & 33 & Licenciatura & 35 & Licenciatura & 18 \\
Maestría & 8 & Maestría & 4 & Maestría & 4 \\
Doctorado & 1 & Doctorado & 7 & Doctorado & 0 \\
Capacitación & & Capacitación & 79 & Capacitación & 36 \\
en servicio & 39 & en servicio & & en servició & \\
Experiencia & 62 & Experiencia & 71 & Experiencia & 87 \\
Intuición & 19 & Intuición & 28 & Intuición & 23 \\
\hline
\end{tabular}

prescripción, referencia a especialistas, administración y práctica general. Esto podría mostrar una fuerte tendencia hacia la educación antes de ejercer, más que la capacitación en servicio. Pero los practicantes de ELT aprenden menos que los demás en la capacitación en servicio, en lo que se refiere a las áreas de conocimiento del área especializada, referencia a especialistas y administración. También aprenden menos por experiencia en las áreas de diagnosis, referencia a especialistas, administración y práctica general. Quizá sorpresivamente, los médicos actúan por intuición más que los demás, y los practicantes de ELT hacen menos uso de la intuición. Esto puede reflejar la necesidad de actuar que tienen la Medicina y Arquitectura, incluso en casos en los que la información disponible es insuficiente para tomar decisiones. De esta manera se explica la necesidad de usar su intuición. Por otro lado, los practicantes de ELT pueden sentirse obligados, por el ambiente académico, a restringir sus acciones a las que pueden ser justificadas por la evidencia.

Otra indicación de la importancia relativa de la educación y experiencia puede ser encontrada en la manera en que los profesionistas son contratados (pregunta P23 en ELT, P20 en Arquitectura y Medicina). En ELT, los dos criterios más citados fueron nombramientos internos basadas en grados académicos (59\%) y nombramientos internos basados en experiencia y referencias (33\%). 
En contraste, en Arquitectura y Medicina los criterios más citados fueron competiciones abiertas y priorizaron la experiencia. En Medicina, la experiencia y referencias fueron un criterio de nombramiento de $92 \%$ de los respondientes, y los grados académicos de $58 \%$, mientras en Arquitectura se dio $70 \%$ y $50 \%$ respectivamente. Esto es consistente con una percepción de ELT como una rama de la actividad académica, pero con la carencia de un reconocimiento académico aceptado en el campo de la enseñanza de idiomas, y puede explicar la predominancia de nombramientos internos, es decir, por evidencias directos del desempeño del maestro.

Aunque la hipótesis está confirmada por los tres grupos de practicantes, los datos muestran que pueden existir diferencias reales y significativas entre ELT y las otras profesiones en cómo adquieren y usan su conocimiento.

Hipótesis 3. Los profesionistas están conscientes de los límites de su conocimiento.

Preguntas de la sección A son dirigidas a descubrir cómo se autoevalúan los encuestados en su nivel de conocimiento del área especializada. Hay tres preguntas en ELT y dos en Medicina y en Arquitectura. En cada profesión una de esas preguntas es acerca de la aplicación práctica del conocimiento, y las otras son acerca del conocimiento teórico. Diferencias entre niveles de conocimiento prácticos y teóricos no son marcadas, entonces los resultados para cada sección pueden ser promediados para facilitar las comparaciones entre las profesiones. Las respuestas en esta sección están anotadas en tal forma que $100 \%$ indicaría que todos los encuestados creen tener todo el conocimiento necesario para hacer su trabajo, mientras una anotación de $20 \%$ indicaría que los encuestados creen que ellos no tiene el suficiente conocimiento para hacer su trabajo. Los promedios anotados son: $74 \%$ para ELT, $83 \%$ para Medicina y $82 \%$ para Arquitectura. Este indicador parece mostrar un reconocimiento saludable de los límites de sus conocimientos en las tres profesiones. ELT muestra ser la más autocrítica. Otro indicador es el reconocimiento de los límites profesionales, el cual se define como la referencia de los clientes a especialistas, donde el conocimiento de los encuestados no es apropiado para las necesidades del cliente.

En la sección $D$ del cuestionario, los encuestados responden a preguntas acerca de referencias a especialistas. El $80 \%$ de los encuestados de ELT afirmaron que ellos en ocasiones enviaron clientes a especialistas, mientras en Medicina 
la cifra fue de $92 \%$ y en Arquitectura de $100 \%$. De nuevo hay una fuerte indicación de reconocimiento de los límites de competencia profesional, y la hipótesis es fuertemente apoyada para las tres profesiones.

Hipótesis 4: Los profesionistas deciden por ellos mismos principalmente, cómo y cuándo hacer su trabajo.

Si se hicieran preguntas generales para esta hipótesis, probablemente se provocaría distorsión en las respuestas debido a los conflictos locales, temporales o por frustraciones con superiores o administradores. Por eso la atención de los encuestados está enfocada en áreas específicas de su trabajo. Hay preguntas acerca de quién tiene la autoridad para decidir cómo y cuando hacer aspectos específicos de su trabajo profesional en secciones B, C, D, E y F de cada cuestionario. El análisis de los resultados por área se muestra en la tabla 3.

Conforme al promedio global, es evidente que ELT no se compara favorablemente con las demás profesiones en el aspecto de autoridad. ELT cae entre la Arquitectura y la Medicina respecto de la autoridad de los practicantes en la prescripción, mientras los resultados de ELT son sustancialmente bajos en las otras áreas. En promedio, los practicantes médicos tienen que aceptar más autoridad de otros, mientras que en Arquitectura hay menor autoridad delegada.

TABLA 3. LA DISTRIBUCIÓN DE AUTORIDAD EN LAS DECISIONES SOBRE CÓMO Y CUÁNDO REALIZAR ACTIVIDADES PROFESIONALES (FRECUENCIA DE REFERENCIA DEL PROFESIONISTA MISMO COMPARADO CON LA OTRA AUTORIDAD MÁS CITADA)

\begin{tabular}{lcccccc}
\hline & \multicolumn{2}{c}{ ELT } & \multicolumn{2}{c}{ Medicina } & \multicolumn{2}{c}{ Arquitectura } \\
& él mismo & otro & él mismo & otro & él mismo & otro \\
& $(\%)$ & $(\%)$ & $(\%)$ & $(\%)$ & $(\%)$ & $(\%)$ \\
\hline B. Diagnosis & 72 & 43 & 83 & 58 & 100 & 10 \\
C. Prescripción & 83 & 37 & 75 & 42 & 100 & 20 \\
$\begin{array}{l}\text { D. Referencia a } \\
\quad \text { especialistas }\end{array}$ & 70 & 18 & 92 & 33 & 100 & 30 \\
$\begin{array}{l}\text { E. Administración } \\
\text { F. Práctica general }\end{array}$ & 67 & 25 & 92 & 42 & 100 & 30 \\
Promedio global & 75 & 32 & 92 & 50 & 100 & 30 \\
\hline & 73 & 31 & 87 & 45 & 100 & 24 \\
\hline
\end{tabular}


TABLA 4. DISTRIBUCIÓN DE AUTORIDAD EN LA EVALUACIÓN DE ACTIVIDADES PROFESIONALES.

(EL PROFESIONISTA MISMO COMPARADO CON LA OTRA AUTORIDAD MÁS CITADA)

\begin{tabular}{llllll}
\hline ELT (P.27) & & \multicolumn{2}{l}{ Medicina (P.24) } & \multicolumn{2}{l}{ Arquitectura(P.24) } \\
\hline Él mismo & otro & Él mismo & otro & Él mismo & otro \\
$56 \%$ & $52 \%$ & $75 \%$ & $67 \%$ & $70 \%$ & $50 \%$ \\
\hline
\end{tabular}

No es sorprendente que los profesionistas, de vez en cuando, tienen que acordar su curso de acción con otros dentro de su organización. La hipótesis es apoyada si los practicantes de manera consistente toman la mayor responsabilidad para decisiones, y ese es el caso para las tres profesiones. Sin embargo, practicantes del ELT aparentan ser menos autónomos que arquitectos y médicos.

Hipótesis 5. Los profesionistas usualmente se evalúan a ellos mismos, como parte de la evaluación de su trabajo que hace un superior.

El profesionalismo está orientado hacia resultados. Por lo tanto es inapropiado evaluar acciones individuales en aislamiento. La evaluación debe ser la entrega global del servicio. La pregunta P27 en el cuestionario de ELT y la pregunta P24 en Arquitectura y Medicina fueron diseñadas para recopilar información para análisis sobre este aspecto. Se esperaría que las organizaciones empleadoras de profesionistas evalúen a su personal profesional, entonces la pregunta aquí es, realmente, sobre cuánto control sienten los profesionistas que tienen en su evaluación. La tabla 4 hace la comparación entre la autoridad ejercida por los propios encuestados y las otras autoridades más citadas en el proceso de evaluación.

Medicina tiene el más alto resultado en auto-evaluación, mientras Arquitectura tiene el más bajo en la evaluación por otros, pero en ambas profesiones la auto-evaluación se menciona más a menudo que la evaluación a través de otros. Por otro lado, en ELT la situación es diferente, la evaluación a través de otros y la auto-evaluación son casi igualmente importantes, y además la auto-evaluación se menciona sustancialmente menos que en Medicina y Arquitectura. Esto es un resultado tal vez sorprendente, dado la 
atención que la enseñanza reflexiva ha recibido en la literatura de ELT (véase Wallace, 1998).

Los resultados apoyan la hipótesis en los casos de Medicina y Arquitectura, pero no en ELT.

Hipótesis 6. Los profesionistas están conscientes de los mecanismos de responsabilidad que operan en su profesión.

En el caso de ELT, como puede observarse en la tabla 4, los practicantes consideran que están evaluados parcialmente por otros, en este caso la institución u organización en la que ellos trabajan (P27). Cualquier procedimiento legal en contra de maestros sería en el campo de abuso de autoridad o negligencia en el cuidado pastoral, y no por deficiencias en el servicio profesional de "enseñar", esto es, hacer posible el aprendizaje. Tales procedimientos serían llevados por la institución contra sus maest-ros siguiendo una demanda por o de parte de los clientes (estudiantes o padres en este caso) en contra de la misma institución. Este tipo de responsabilidad cae fuera del alcance de este estudio, pero sería interesante saber si los practicantes entienden su posición. En ELT, la pregunta 28 es sobre qué prácticas consideran el criterio principal para su evaluación. Un $89 \%$ de los encuestados mencionaron la opinión de los estudiantes, $61 \%$ mencionaron los resultados de los estudiantes, y $45 \%$ mencionaron mantenerse al corriente en conocimientos de ELT. Esta distribución puede reflejar los intereses de la institución, porque aunque la opinión del cliente es importante, los encuestados mencionaron con menor frecuencia los resultados de los estudiantes como un criterio de evaluación. Pero tal vez este último puede ser mejor indicador de servicio para el clienteestudiante que la opinión subjetiva del cliente. La información al respecto aún es insuficiente, pero nos deja un tema de gran interés para futuras investigaciones, para qué y para quién trabajan los profesionales de la educación. En la comparación de los resultados de esta hipótesis, 100\% de los arquitectos y $58 \%$ de los médicos fueron evaluados en opinión de los clientes, $90 \%$ de los arquitectos y $25 \%$ de los médicos fueron evaluados por resultados, y $30 \%$ de los arquitectos y $68 \%$ de los médicos fueron evaluados por mantenerse al corriente.

En los cuestionarios de Medicina y Arquitectura, se pregunta a los encuestados qué tan de acuerdo están con cada una de las declaraciones 
acerca de su responsabilidad (PI7 en Medicina y Arquitectura, P22 en ELT). Las respuestas a esas preguntas están calificadas de tal manera que el $60 \%$ indicaría una posición no comprometida, mientras $100 \%$ indicaría un completo acuerdo entre los encuestados.

La aclaración "Mi trabajo es tener la responsabilidad legal para un servicio profesional............. de diagnosis, prescripción, referencia a especialistas y documentación del servicio" produjo una cifra de $80 \%$ de acuerdo en Medicina y $82 \%$ de acuerdo en Arquitectura. Estos resultados indican que ambos practicantes están de acuerdo con el modelo de servicio profesional propuesto en este estudio y que ellos son legalmente responsables por entregarlo, apoyando la hipótesis en los casos de Medicina y Arquitectura. La misma pregunta, pero sin referencia a la responsabilidad legal, resultó en una cifra de solamente $60 \%$ en ELT.

Una declaración secundaria "Estoy dispuesto a aceptar mi responsabilidad legal por el privilegio de ejercer mi ........ habilidad y juicio" puede dar una visión de la percepción de los practicantes de la justicia de las responsabilidades legales que asumen los profesionistas. Practicantes médicos están de acuerdo con una cifra de $87 \%$, mientras los arquitectos fueron ligeramente entusiastas con $78 \%$ reflejando quizás la naturaleza más litigiosa de sus clientes. Los practicantes de ELT reportaron $56 \%$ de acuerdo, representando una visión sustancialmente diferente de la responsabilidad legal personal.

La hipótesis esta apoyada por arquitectos y médicos, pero no por ELT.

Hipótesis 7. Los profesionistas principalmente disfrutan su trabajo a pesar de frustraciones y carencia de reconocimiento.

Los encuestados fueron cuestionados qué tan de acuerdo están con la declaración "Mi trabajo es agradable" (PI7 en Medicina y Arquitectura, P22 en ELT). Las respuestas a esa declaración están calificados de tal manera que $60 \%$ indicaría una posición no comprometida, mientras 100\% indicaría entero acuerdo por todos los encuestados.

Las respuestas en ELT fueron $92 \%$, en Medicina $85 \%$ y Arquitectura $82 \%$. Esos son resultados altos y apoyan la hipótesis por las tres profesiones estudiadas.

En las tres profesiones los encuestados reportaron problemas con carencia de posición social y reconocimiento por su trabajo. Son problemas 
que hacen a las personas infelices con su trabajo normalmente, aunque en este estudio no es el caso. Tal vez está demostrando un fuerte sentido de vocación en las tres profesiones.

Hipótesis 8. Los profesionistas ven su trabajo principalmente como un servicio al cliente.

Los encuestados fueron cuestionados sobre qué tan de acuerdo estaban con la afirmación "Mi trabajo es ayudar personas con los problemas de. que surgen de su......" donde el tipo de problema apropiado para cada profesión está sustituida en el primer espacio y la necesidad apropiada del cliente en el segundo (P22 en ELT, PI7 en Medicina y Arquitectura). Las respuestas a estas afirmaciones se calculan de tal manera que $60 \%$ indicaría una posición no comprometida, mientras $100 \%$ indicaría un completo acuerdo de todos los encuestados. Los resultados registrados fueron: $81 \%$ para ELT, $85 \%$ para Medicina y $92 \%$ para Arquitectura. Esos son resultados de porcentajes altos y sugieren un sentido de servicio.

Una segunda declaración en la misma pregunta cuestiona si los encuestados están de acuerdo con "La principal compensación para mi trabajo es el privilegio de ayudar a alguien." Los encuestados en ELT reportaron $83 \%$ de acuerdo, los practicantes médicos $70 \%$ y los arquitectos $60 \%$. Las respuestas menos entusiastas de los médicos y especialmente los arquitectos podrían explicarse parcialmente a los salarios relativamente altos que ellos disfrutan comparados con los practicantes ELT. En Medicina el salario es mencionado en segundo lugar con $68 \%$, mientras en Arquitectura el salario está en primer lugar con $62 \%$. No obstante, de las tres afirmaciones sobre compensación por el servicio profesional, cada profesión calificó el privilegio de ayudar a otros por encima de la posición social.

Hay apoyo para la hipótesis en los resultados de las tres profesiones.

Hipótesis 9. Los profesionistas necesitan actualizar su conocimiento profesional rápidamente pero son cuidadosos sobre la aplicación de nuevas ideas.

El desarrollo profesional continuo es uno de los temas de la sección $G$ del cuestionario. En ELT se mencionó que la forma principal de mantenerse al corriente es tomando cursos. El $86 \%$ de los encuestados contestó que toma 
cursos, $80 \%$ asiste a conferencias, $71 \%$ lee libros sobre ELT y el $36 \%$ lee revistas especializadas (P25). En Medicina, 100\% de los encuestados se mantienen al corriente leyendo revistas especializadas y tomando cursos, seguido por $92 \%$ que asiste a conferencias y $75 \%$ lee libros (P22). En Arquitectura 100\% de los encuestados se mantienen al corriente leyendo revistas especializadas, $70 \%$ fueron a cursos y $40 \%$ leen libros (P22).

Todos los encuestados hicieron algo para mantenerse al corriente, pero el papel de las revistas especializadas es notablemente diferente en ELT, en comparación con Medicina y Arquitectura. Esto apoya dos ideas relacionadas. Parece que ELT puede ser manejada por teoría en lugar de la aplicación del conocimiento actual. Como evidencia está el bajo uso de revistas especializadas, la forma más rápida de comunicación, y una preferencia por los libros que es el medio más indicado para exponer en extenso una posición teórica. Por otro lado, poner al corriente un servicio profesional es indispensable, y requiere conocimiento actualizado a través de las revistas especializadas.

Tabla 5. Evaluación de nUeVAs IDEAS.

\begin{tabular}{lccc}
\hline & $\begin{array}{c}\text { ELT } \\
(\%)\end{array}$ & $\begin{array}{c}\text { Medicina } \\
(\%)\end{array}$ & $\begin{array}{c}\text { Arquitectura } \\
(\%)\end{array}$ \\
\hline Las prueba & 91 & 83 & 50 \\
Las discute con & 64 & 100 & 100 \\
sus colegas & & & \\
Lee más & 60 & 100 & 60 \\
Otro & 2 & 0 & 10 \\
\hline
\end{tabular}

En ELT, 91\% de los practicantes evaluaron nuevas ideas por la aplicación de éstas, mientras en Medicina y Arquitectura las cifras son $83 \%$ y $50 \%$ respectivamente. La diferencia entre las cifras para ELT por un lado y de Arquitectura por otro, puede reflejar diferencias reales en actitud. El deseo de los practicantes de ELT por probar nuevas ideas es enteramente consistente con un espíritu de investigación académica, pero no con la precaución apropiada para un profesional consciente de que los clientes pueden ser perjudicados o ayudados por nuevas ideas. El alto resultado en Medicina es 
quizás sorprendente, pero los practicantes siempre reportaron discutir el asunto con sus colegas y siempre leen más, sugiriendo una precaución adecuada. Solamente $64 \%$ de los practicantes de ELT reportaron discutir nuevas ideas con colegas, mientras todos los arquitectos y médicos lo hacen.

En este caso, la hipótesis es apoyada por los resultados de Medicina y Arquitectura, pero no por ELT.

Hipótesis 10. ELT aún no conforma una conceptualización profesional del servicio en algunos aspectos.

En este estudio se ha tratado de medir hasta qué punto las profesiones establecidas pueden conceptuar su trabajo dentro de una estructura desarrollada para la emergente profesión de ELT. El análisis de los resultados con relación a la Hipótesis I sugiere que la estructura se puede aplicar en varias profesiones, pero la práctica actual de ELT no se conforma al modelo.

Dentro del modelo hay detalles que buscan dar más precisión a la forma en que puede ser adaptado para cada profesión, y algunas veces causan ambigüedades, los cuales han sido tratados en el transcurso de este estudio.

Otros detalles pueden mostrar puntos de acuerdo entre diferentes profesiones, mientras no sean criterios para distinguir profesiones de otras industrias de servicios. Entre esos por ejemplo, pueden caer repuestas a las afirmaciones "Mi trabajo es desafiante" en la pregunta P22 en ELT y 17 en Medicina y Arquitectura. Se puede esperar que la respuesta fuera afirmativa porque sería preocupante encontrar que algún profesional pensara que su trabajo fuera fácil por un lado, o demasiado difícil por el otro. Las respuestas de las tres profesiones muestran $90 \%$ de acuerdo, donde las respuestas están analizadas de tal manera que $60 \%$ indicaría una posición no comprometida, mientras $100 \%$ indicaría un completo acuerdo por todos los encuestados. Aunque las tres profesiones son similares en esto, no marca una diferencia de otros trabajos que pueden también ser desafiantes. De igual manera, las tres profesiones se quejan de carencia o desgaste de posición social y carencia de apreciación de su trabajo (P28 en ELT, 26 en Medicina y Arquitectura) pero en la realidad hay pocos trabajos que son evaluados como los trabajadores desearían.

La tabla 6 muestra la homogeneidad de profesiones en las diferentes partes de su servicio. Quizá no se encuentra unanimidad en ELT debido al relativo 
TABLA 6. Cosas QUE HACEN TODOS LOS PROFESIONISTAS.

\begin{tabular}{|c|c|c|c|}
\hline & ELT & Medicina & Arquitectura \\
\hline $\begin{array}{l}\text { A) Conocimiento } \\
\text { del área } \\
\text { especialista }\end{array}$ & & & $\begin{array}{l}\text { - Aprende sobre la construcción } \\
\text { práctica en más de una manera } \\
\text { (P4) }\end{array}$ \\
\hline B) Diagnosis & & & $\begin{array}{l}\text { - Evalúa resultados para las } \\
\text { decisiones del diseño (P5) } \\
\text { - Decide cómo y cuando (P7) }\end{array}$ \\
\hline C) Prescripción & & & $\begin{array}{l}\text { - Ayuda a los clientes a elegir } \\
\text { opciones de diseño (P8) } \\
\text { - Hace elecciones de diseño (P) } \\
\text { - Decide cómo y cuando (PIO) }\end{array}$ \\
\hline $\begin{array}{l}\text { D) Referencia a } \\
\text { especialistas }\end{array}$ & & & $\begin{array}{l}\text { - Ayudan a los clientes escoger a } \\
\text { especialistas adecuadas para } \\
\text { sus necesidades (PII) } \\
\text { - Aprenden a hacerlo a través de } \\
\text { experiencia (PI2) } \\
\text { - Decide cómo y cuando (PI3) }\end{array}$ \\
\hline E) Administración & & $\begin{array}{l}\text { - Guarda archivos de } \\
\text { los detalles médicos } \\
\text { de pacientes (PI4) }\end{array}$ & $\begin{array}{l}\text { - Guarda archivos de las instruc- } \\
\text { ciones de clientes (PI4) } \\
\text { - Guarda archivos de progreso } \\
\text { de la construcción (PI4) } \\
\text { - Guarda archivos de } \\
\text { decisiones del cliente (PI4) } \\
\text { - Decide cómo y cuando (PI6) }\end{array}$ \\
\hline F) Práctica general & & & $\begin{array}{l}\text { - Decide qué metodología usar } \\
(\mathrm{PI} 9) \\
\text { - Aprende a hacerla a través de } \\
\text { experiencia (PI8) }\end{array}$ \\
\hline $\begin{array}{l}\text { G) Desarrollo } \\
\text { profesional }\end{array}$ & & $\begin{array}{l}\text { - Lee revistas (P22) } \\
\text { - Va a cursos(P22) } \\
\text { - Discute nuevas ideas } \\
\text { con colegas (P23) } \\
\text { - Lee más sobre nuevas } \\
\text { ideas(P23) }\end{array}$ & $\begin{array}{l}\text { - Lee revistas (P22) } \\
\text { - Discute nuevas ideas con } \\
\text { colegas (P23) } \\
\text { - Evaluado por opinión del cliente } \\
\text { (P25) }\end{array}$ \\
\hline
\end{tabular}


tamaño grande de la muestra, pero las cosas en las que los arquitectos y médicos están de acuerdo es interesante en el contexto de la discusión anterior.

\section{Conclusiones}

Este estudio se llevó a cabo con muestras pequeñas de dos profesiones, Arquitectura y Medicina en el Reino Unido, como punto de referencia para el profesionalismo relativo de una muestra de practicantes de ELT en México. Los principales resultados se resumen a continuación:

Hipótesis I. Los profesionistas pueden concebir su trabajo dentro de la estructura sugerida.

Hipótesis confirmada en Arquitectura y Medicina, quedó en duda en ELT.

Hipótesis 2. Los profesionistas usualmente aprenden a hacer su trabajo en estudios de licenciatura en cualquier área del conocimiento, estudiando por su cuenta y experiencia práctica, pero no por estudiar posgrados.

Hipótesis confirmada, aunque los practicantes de ELT dieron mayor importancia a la capacitación en pre-servicio a diferencia de los arquitectos y médicos que le dieron mayor importancia a la capacitación en servicio.

Hipótesis 3. Los profesionistas están conscientes de los límites de su conocimiento. Hipótesis confirmada en Arquitectura, Medicina y ELT.

Hipótesis 4. Los profesionistas deciden por ellos mismos principalmente, cómo y cuándo hacer su trabajo.

Hipótesis confirmada en Arquitectura, Medicina y ELT.

Hipótesis 5. Los profesionistas usualmente se evalúan a ellos mismos, como parte de la evaluación de su trabajo que hace un superior.

Hipótesis conformada en Medicina y rechazada en ELT. Se encontró que la muestra de los practicantes de ELT fueron evaluados predominantemente por otros, en contraste a las otras dos profesiones quienes vieron la autoevaluación como una parte importante de su evaluación. 
Hipótesis 6. Los profesionistas están conscientes de los mecanismos de responsabilidad que operan en su profesión.

Hipótesis confirmada en Arquitectura y Medicina, en duda en eLT. EI instrumento no proporcionó datos para ELT y es evidente que en el marco legal y la práctica laboral ELT es diferente, en este aspecto, en comparación con Medicina y Arquitectura.

Hipótesis 7. Los profesionistas principalmente disfrutan su trabajo a pesar de frustraciones y carencia de reconocimiento.

Hipótesis confirmada en Arquitectura, Medicina y ELT.

Hipótesis 8. Los profesionistas ven su trabajo principalmente como un servicio al cliente.

Hipótesis confirmada en Arquitectura, Medicina y ELT.

Hipótesis 9. Los profesionistas necesitan actualizar su conocimiento profesional rápidamente pero son cuidadosos sobre la aplicación de nuevas ideas.

Hipótesis confirmada en Arquitectura, Medicina y rechazada en ELT. La práctica de ELT parece estar más validada por un espíritu académico de búsqueda que en la atención a las necesidades del cliente.

Hipótesis 10. ELT aún no conforma una conceptualización profesional del servicio en algunos aspectos.

Hipótesis confirmada: en ELT muestra resultados diferentes a la hipótesis 2, 5, y 9 y no se muestra su comprobación en las hipótesis 1 y 6.

Hay una indicación global que ELT puede ser vista por sus practicantes como una actividad especializada proyectada a traer beneficios a los estudiantes, pero a través de la excelencia de los maestros en lugar de a través de los servicios a los estudiantes. Esta distinción es importante en la conceptualización del profesionalismo, para que el perfil del practicante de ELT se acerque al del artista y al académico, más que al experto y proveedor de servicio responsable, pero sin mucha selección rigurosa y entrenamiento del artista o los efectos inocuos de la investigación académica. No obstante, a pesar de la ausencia de una organización profesional, los practicantes de ELT 
están cerca, en muchos aspectos de su trabajo práctico, de las profesiones de referencia, es decir, Medicina y Arquitectura en el Reino Unido.

Este estudio presenta los resultados de un proyecto a largo plazo en el que una muestra más grande de respuestas de arquitectos y médicos será recolectada para hacer un análisis más profundo sobre las conclusiones alcanzadas hasta ahora.

\section{Fuentes Consultadas}

Abbott, A. (1988). The System of Professions: An Essay on the Division of Expert Labor. Chicago: University of Chicago Press.

Dingwall, R. y P. Fenn. (1987). "A respectable profession? Sociological and economic perspectives on the regulation of professional services" International Review of Law and Economics, 7, 5I-64.

Freidson, E. (200I). Professionalism, the Third Logic: on the Practice of Knowledge. Chicago: University of Chicago Press.

Goode,W.J.(1969).“The theoretical limits of professionalization” en A. Etzione (ed.) The Semi- Professions and their Organization. Nueva York: The Free Press.

Illich, I. et al. (1977). Disabling Professions. Nueva York: Marion Boyars.

Larson, M. S. (1977). The Rise of Professionalism: a Sociological Analysis. Berkeley: University of California Press.

Wallace, M. J. (1998). Action Research for Language Teachers. Cambridge: Cambridge University Press. 


\section{ApÉndice}

\section{Participantes}

INSTITUCIÓN/PRÁCTICA NÚMERO DE PARTICIPANTES

$\begin{array}{rc}\begin{array}{r}\text { Capita Symonds, Lancaster and Kendal } \\ \text { Harrison Pitt Architects, Lancaster }\end{array} & 3 \text { arquitectos } \\ \text { I arquitecto } \\ \text { Jhomas Associates Architects, Lancaster } & \text { I arquitecto } \\ \text { JMP Architects, Lancaster } & 4 \text { arquitectos } \\ \text { PJB Chartered Architects, Colchester } & \text { I arquitecto } \\ \text { Queen Square Practice, Lancaster } & 4 \text { médicos } \\ \text { Dr. Mary Muir and colleagues, Aberdeen } & 7 \text { médicos } \\ \text { Rowhedge Surgery, Colchester } & \text { I médico } \\ \text { Universidad de la Sierra, Oaxaca, México } & \text { I maestro } \\ \text { IPN, Zacatenco y Santo Tomás } & 57 \text { maestros } \\ \text { British Council, México D. F. } & 7 \text { maestros } \\ \text { Asistentes en ANUPI, Puebla, 2004 } & 13 \text { maestros } \\ \text { Uaemx, Estado de México } & 29 \text { maestros }\end{array}$

\section{Instrumentos y tablas de resultados}

\section{Professionalism Questionnaire Results} Architects in the UK

Personal Details:

Are you an Architect registered with the Architects Registration Board? $100 \%$ confirmed that they are.

Do you work

In public service?

In private practice?

$100 \%$

As a sole practitioner?

$0 \%$ 
Profesionalismo en la enseñanza del idioma inglés (ELT)

Job Details

Have responsibility for other Architects? $\quad 70 \%$

Teach?

$10 \%$

Do research?

$20 \%$

Have a speciality?

$20 \%$

A) Technical KNoWledge

I. How do you rate your theoretical knowledge of building? $84 \%$

2. How did you learn about theory of building? $\mathbf{9 0 \%}$ of respondents indicated more than one option.

\begin{tabular}{lr} 
Self study & $50 \%$ \\
Diploma & $60 \%$ \\
Bachelor's & $90 \%$ \\
Master's & $10 \%$ \\
Doctorate & $0 \%$ \\
In- service training & $60 \%$ \\
Experience & $90 \%$ \\
Intuition & $10 \%$ \\
Continuing professional & \\
\multicolumn{2}{l}{ development }
\end{tabular}

3. How do you rate your practical knowledge of building? $\mathbf{8 0 \%}$

4. How did you learn about practical building? $100 \%$ of respondents indicated more than one option

Self study

Diploma

Batchelor's

Master's

Doctorate

In- service training

Experience

Intuition
$50 \%$

$40 \%$

$40 \%$

$0 \%$

$0 \%$

$50 \%$

$10 \%$

$0 \%$ 
B) DIAGNOSIS

5. Do you ever do any of the following in your job as an Architect?

Evaluate outcomes for design decisions

Evaluate clients' decisions

Evaluate clients' decision making ability

Identify clients' decision making style
$100 \%$

$90 \%$

$80 \%$

$60 \%$

6. How did you learn to do these activities? $80 \%$ of respondents indicated more than one option

$\begin{array}{lr}\text { Self study } & 50 \% \\ \text { Diploma } & 20 \% \\ \text { Bachelor's } & 0 \% \\ \text { Master's } & 0 \% \\ \text { Doctorate } & 0 \% \\ \text { In- service training } & 20 \% \\ \text { Experience } & 90 \% \\ \text { Intuition } & 40 \%\end{array}$

7. Who decides how and when you should do these activities?

You

Your head of department/area

Your work organization
$100 \%$

$10 \%$

$10 \%$

C) Prescription

8. Do you ever do any of the following in your job as an Architect?

Help clients to make design choices

$100 \%$

Make design choices

$100 \%$

Plan outcomes, costs and timescales

$90 \%$

9. How did you learn to do these activities? $\mathbf{7 0 \%}$ of respondents indicated more than one option.

Self study

Diploma
$40 \%$

$20 \%$ 


$\begin{array}{lr}\text { Bachelor's } & 10 \% \\ \text { Master's } & 10 \% \\ \text { Doctorate } & 0 \% \\ \text { In-service training } & 30 \% \\ \text { Experience } & 90 \% \\ \text { Intuition } & 30 \%\end{array}$

10. Who decides how and when you should do these activities? $20 \%$ of respondents indicated more than one option

You

Your head of department/area

Your work organization
$100 \%$

$0 \%$

$20 \%$

D) Referral to Specialists

II. Do you ever do any of the following in your job as an Architect?

Help clients to choose specialists suited to their needs $100 \%$ Help clients to choose technology suited to their needs $90 \%$

12. How did you learn to do these activities? $\mathbf{6 0 \%}$ of respondents indicated more than one option

Self study

Diploma

Bachelor's

Master's

Doctorate

In-service training

Experience

Intuition

Continuing professional

development
$20 \%$

$10 \%$

$10 \%$

$0 \%$

$0 \%$

$40 \%$

$100 \%$

$20 \%$

$10 \%$

13. Who decides how and when you should do these activities? $30 \%$ of respondents indicated more than one option

You $\quad 100 \%$ 
Your head of department/area $\quad 0 \%$

Your work organization $\quad 30 \%$

The client

E) Administration

14. Do you ever do any of the following in your job as an Architect?

Keep records of clients' briefs

Keep records of building progress

Keep records of clients' decisions

Keep records of clients' attitude to the project

Keep records of referrals to other specialists or technologies

Advise clients in writing of any of the above
$100 \%$

$100 \%$

$100 \%$

$60 \%$

$90 \%$

$90 \%$

15. How did you learn to do these activities? $50 \%$ of respondents indicated more than one option

Self study

Diploma

Bachelor's

Master's

Doctorate

In-service training

Experience

Intuition
$30 \%$

$10 \%$

$10 \%$

$10 \%$

$0 \%$

$40 \%$

$90 \%$

$10 \%$

16. Who decides how and when you should do these activities? $30 \%$ of respondents indicated more than one option

You

Your head of department/area

Your work organization

The client
$100 \%$

$10 \%$

$30 \%$

$10 \%$ 
F) General Practice

17. How would you classify your approach to Architecture?

My job is enjoyable

My job is challenging

My job is helping people with design and technical problems that arise from their building needs

My job is taking legal responsibility for a professional architectural service of diagnosis, prescription, referral and record keeping.

My main compensation for my job is the privilege of knowing I have helped someone

My main compensation for my job is the privilege of having status in the community

My main compensation for my job is the salary I receive

I am willing to accept my legal liability for the privilege of exercising my architectural skill and judgment
$92 \%$ $82 \%$

$60 \%$

$82 \%$

$90 \%$

$82 \%$

$62 \%$

$78 \%$

18. How did you learn these approach(es)? $\mathbf{9 0 \%}$ of respondents indicated more than one option.

Self study

Diploma

Bachelor's

Master's

Doctorate

In-service training

Experience

Intuition
$20 \%$

$20 \%$

$10 \%$

$0 \%$

$0 \%$

$30 \%$

$100 \%$

$30 \%$ 
19. Who decides which approach(es) to use $\mathbf{3 0} \%$ of respondents indicated more than one option

You

$100 \%$

Your head of department/area

$0 \%$

Your work organization

G) Professional Development

20. How did you get your job as an Architect?

Internal appointment based on experience and references $20 \%$

Internal appointment based on qualifications

$10 \%$

Open competition based on experience and references $70 \%$

Open competition based

on qualifications

$50 \%$

Other

Self employed

$10 \%$

2I. Who made the decision about your appointment?
An Architect
$90 \%$
An Technical committee
$0 \%$
An Administrator
$0 \%$

22. How do you keep up to date with new ideas?

Reading books on Architecture

$40 \%$

Reading journals on Architecture $\quad 100 \%$

Going on courses

$70 \%$

Going to conferences

$40 \%$

Other

Travelling $\quad 10 \%$

CPD

$10 \%$ 
23. How do you evaluate new ideas?

Try them out $\quad 50 \%$

$62 \quad$ Discuss them with colleagues $100 \%$

Read more about them $\quad 60 \%$

Other

Theoretical evaluation before trying them 10\%

24. Who evaluates your work as an Architect?

You $\quad 70 \%$

Your head of department/area $\quad 40 \%$

An technical committee $\quad 0 \%$

Your work organization $\quad 50 \%$

Clients 20\%

Building users $\quad 10 \%$

25. How are you evaluated?

Clients' opinions 100\%

Building completion $\quad 90 \%$

Number of clients $\quad 30 \%$

Total construction value $\quad 40 \%$

Research and publication $0 \%$

Keeping up to date $\quad 30 \%$

Participation in committees $\quad 0 \%$

Contribution to society generally $\quad 10 \%$

Performance generally in successfully

completing projects and the work leading up to that. Competency in dealing with all aspects of a project.

Contribution to and ability to work in a team

Completion of projects on time \& budget, Design Awards $\quad 10 \%$

Cost certainty 
26. What do you think of your work as an Architect and your status in your institution?

'Resolved, status is irrelevant.' (I)

'Status is not particularly high- architects seem to be scapegoats and not well regarded.' (3)

'It is rewarding, challenging, frustrating, but always varied, never boring.' (4)

'It is often challenging, occasionally rewarding, constantly changing.' (5)

'I enjoy my work (on the whole) and I cannot imagine many others careers which would give me the satisfaction completed job - something which looks good, works well and meets or exceeds the client's expectations. I think I am good at my job (though I am always aware that there is always scope for improving the service provided in however small a manner). I think I am well regarded in my place of work.' (6)

'An exposed (in terms of litigation) and generally under remuneratedprofession.' (7)

'I am not satisfied with it and don't believe Architecture is valued by my employer.' (8)

'Work is very enjoyable, status is not issue.' (9)

'Architecture does not necessarily give status in society, It is an interesting, fulfilling job, but we are often no longer the lead consultant in the building procurement process. Accountant lead issues often dictate more than us. We are however very important as the coordinator of all aspects of the process.' (10) 


\section{Professionalism Questionnaire Results}

\section{Medical Doctors in the UK}

Personal Details

Are you a medical practitioner registered with the General Medical Council? $100 \%$ confirmed that they are.

Do you work

In public service?

$100 \%$

In private practice?

$0 \%$

As a sole practitioner? $\quad 0 \%$

Job Details

Have responsibility for other Doctors? 67\%

Teach?

Do research?

Have a speciality?
$83 \%$

$17 \%$

$67 \%$

\section{A) Medical Knowledge}

I. How do you rate your knowledge of Medicine in your field? 83.33\%

2. How did you learn Medicine? $\mathbf{7 5 \%}$ of respondents indicated more than one option.

$\begin{array}{lr}\text { Self study } & 42 \% \\ \text { Diploma } & 8 \% \\ \text { Bachelor's } & 92 \% \\ \text { Master's } & 17 \% \\ \text { Doctorate } & 8 \% \\ \text { In-service training } & 50 \% \\ \text { Experience } & 58 \% \\ \text { Intuition } & 25 \%\end{array}$

3. How do you rate your knowledge of the application of Medicine in your field? $83.33 \%$ 
4. How did you learn about the practical application of Medicine? 75\% of respondents indicated more than one option.

$\begin{array}{lr}\text { Self study } & 50 \% \\ \text { Diploma } & 8 \% \\ \text { Bachelor's } & 50 \% \\ \text { Master's } & 8 \% \\ \text { Doctorate } & 0 \% \\ \text { In-service training } & 75 \% \\ \text { Experience } & 75 \% \\ \text { Intuition } & 25 \%\end{array}$

B) DIAGNOSIS

5. Do you ever do any of the following in your job as a Doctor?

Evaluate outcomes for patients' treatments $\quad 83 \%$

Evaluate patients' progres $\quad 92 \%$

Evaluate patients' contribution to treatment $58 \%$

Identify patients' medical condition $\quad 92 \%$

6. How did you learn to do these activities? $\mathbf{9 2} \%$ of respondents indicated more than one option.

$\begin{array}{lr}\text { Self study } & 50 \% \\ \text { Diploma } & 17 \% \\ \text { Bachelor's } & 33 \% \\ \text { Master's } & 8 \% \\ \text { Doctorate } & 8 \% \\ \text { In-service training } & 83 \% \\ \text { Experience } & 75 \% \\ \text { Intuition } & 33 \%\end{array}$

7. Who decides how and when you should do these activities? $\mathbf{5 0 \%}$ of respondents indicated more than one option

You

Your head of department/area

Your institution
$83 \%$

$33 \%$

$58 \%$ 
C) Prescription

8. Do you ever do any of the following in your job as a Doctor?

Help patients to make choices about their treatment $\quad 83 \%$ Choose treatments

Plan treatment outcomes, costs and timescales

9. How did you learn to do these activities? $\mathbf{5 8 \%}$ of respondents indicated more than one option

Self study $\quad 58 \%$

Diploma

Bachelor's

Master's

Doctorate

In-service training

Experience

Intuition
$8 \%$

$33 \%$

$0 \%$

$8 \%$

$58 \%$

$58 \%$

$17 \%$

10. Who decides how and when you should do these activities

$\begin{array}{ll}\text { You } & 75 \% \\ \text { Your head of department/area } & 25 \% \\ \text { Your work organization } & 42 \%\end{array}$

D) Referral to Specialists

II. Do you ever do any of the following in your job as a Doctor?

Help patients to choose specialists suited to their condition $50 \%$ Help patients to choose clinics suited to their condition $\quad 50 \%$ Help patients to choose treatments suited to their condition $75 \%$

12. How did you learn to do these activities? $83 \%$ of respondents indicated more than one option.

$\begin{array}{ll}\text { Self study } & 50 \% \\ \text { Diploma } & 17 \% \\ \text { Bachelor's } & 25 \%\end{array}$


Master's $\quad 0 \%$

Doctorate $\quad 8 \%$

In-service training $\quad 83 \%$

Experience $\quad 75 \%$

Intuition

$25 \%$

13. Who decides how and when you should do these activities?

$\begin{array}{ll}\text { You } & 92 \% \\ \text { Your head of department/area } & 25 \% \\ \text { Your work organization } & 33 \%\end{array}$

E) Administration

14. Do you ever do any of the following in your job as a Doctor?

Keep records of patients' medical condition $100 \%$

Keep records of patients' progress $\quad 83 \%$

Keep records of patients' contribution to their treatment $67 \%$

Keep records of patients' attitude to their treatment $\quad 67 \%$

Keep records of referrals to other specialists, clinics

or treatments $\quad 92 \%$

Advise patients in writing of any of the above $\quad 67 \%$

15. How did you learn to do these activities? $\mathbf{7 5 \%}$ of respondents indicated more than one option.

$\begin{array}{lr}\text { Self study } & 58 \% \\ \text { Diploma } & 0 \% \\ \text { Bachelor's } & 33 \% \\ \text { Master's } & 0 \% \\ \text { Doctorate } & 8 \% \\ \text { In-service training } & 92 \% \\ \text { Experience } & 75 \% \\ \text { Intuition } & 33 \%\end{array}$

16. Who decides how and when you should do these activities? $42 \%$ of respondents indicated more than one option. 
You

Your head of department/area

Your work organization
$92 \%$

$25 \%$

$42 \%$

\section{F) General Practice}

17. How would you classify your approach to Medicine (Circle your opinion about the following statements on a scale of $\mathrm{I}-5$, where $\mathrm{I}=$ disagree strongly and 5 = agree strongly)

My job is enjoyable

$85 \%$

My job is challenging $90 \%$

My job is helping people with the medical and emotional problems that arise from their condition $85 \%$

My job is taking legal responsibility for a professional medical service of diagnosis, prescription, referral and record keeping. $80 \%$

My main compensation for my job is the privilege of knowing I have helped someone. $\quad 70 \%$

My main compensation for my job is the privilege of having status in the community $\quad 50 \%$

My main compensation for my job is the salary I receive

I am willing to accept my legal liability for the privilege of exercising my medical skill and judgment.

$86.66 \%$

18. How did you learn your approach? 75\%of respondents indicated more than one option

$\begin{array}{lr}\text { Self study } & 58 \% \\ \text { Diploma } & 17 \% \\ \text { Bachelor's } & 17 \% \\ \text { Master's } & 0 \% \\ \text { Doctorate } & 8 \% \\ \text { In-service training } & 92 \% \\ \text { Experience } & 75 \%\end{array}$


Intuition

19. Who decides which approach(es) to use? $50 \%$ of respondents indicated more than one option

You

$92 \%$

Your head of department/area

$25 \%$

Your work organization

$50 \%$

\section{G) Professional Development}

20. How did you get your job as an Doctor

Internal appointment based on experience and references $\quad 0 \%$ Internal appointment based on qualifications

$0 \%$

Open competition based on experience and references

$92 \%$

Open competition based on qualifications

$58 \%$

2I. Who made the decision about your appointment?
A Doctor
$42 \%$
A Medical committee
$67 \%$
An Administrator
$25 \%$
An appointment committee
$17 \%$

22. How do you keep up to date with new ideas?

$\begin{array}{ll}\text { Reading books on Medicine } & 75 \% \\ \text { Reading journals on Medicine } & 100 \% \\ \text { Going on courses } & 100 \% \\ \text { Going to conferences } & 92 \% \\ \text { Internet } & 33 \% \\ \text { Discussions with colleagues } & 8 \%\end{array}$

23. How do you evaluate new ideas?

Try them out

$83 \%$

Discuss them with colleagues

Read more about them
$100 \%$

$100 \%$ 
24. Who evaluates your work as a Doctor?

$\begin{array}{lr}\text { You } & 75 \% \\ \text { Your head of department/area } & 17 \% \\ \text { A Medical committee } & 0 \% \\ \text { Your work organization } & 67 \% \\ \text { Colleagues } & 8 \%\end{array}$

25. How are you evaluated?

$\begin{array}{lr}\text { Patients' opinions } & 58 \% \\ \text { Patients' recovery } & 25 \% \\ \text { Treatment load- Number of patients } & 33 \% \\ \text { Administrative load } & 33 \% \\ \text { Research and publication } & 33 \% \\ \text { Keeping up to date } & 67 \% \\ \text { Participation in institutional committees } & 25 \% \\ \text { Contribution to society generally } & 8 \% \\ \text { Objective } & 8 \%\end{array}$

26. What do you think of your work as a Doctor and your status in your institution?

'I think the work is a privilege, a challenge, of infinite variety, and significant status in society. I enjoy my work but the pressures of administration are increasing and encroaching on clinical work.' (I)

'Often enjoyable and fulfilling but can also be very stressful. Status is changing. At times it seems that we are open to much criticism but because of patient confidentiality have little redress. At other times I feel my patients value the work I do.' (2)

'It is probably a better job than many. High status.' (3)

'I love my work. I don't care about status- it gets in the way.' (4)

'My work is rewarding, my status is not an issue.' (8)

'Quite happy with both.' (9)

'Hard and challenging, but interesting and rewarding. Status good.' (I I) 'Not bad!' (12) 


\section{Professionalism Questionnaire Results}

ELT in Mexico

Personal Details

Are you a qualified English Teacher? $\mathbf{6 2 . 2 6 \%}$ confirmed that they are, in their opinion.

State your qualifications: $\mathbf{4 . 7 1 \%}$ gave Masters' degrees as their highest academic qualification, 14.1 5\% Batchelor's, 15.09\% COTE , 15.09\% Diploma, 0.94\% Doctorate, $\mathbf{4 . 7}$ I\% Teacher's, I.80\% Courses CAE, $\mathbf{4 4 . 3 3 \%}$ not stated TOTAL: $100 \%$

Employment:

In a public university?

$81.13 \%$

In a private university?

$17.92 \%$

As a sole practitioner?

$14.15 \%$

In British council?

$1.80 \%$

In a language school?

$1.80 \%$

Job Details

Responsibility

Have responsibility for other English Teacher?

$17.92 \%$

Teach?

$93.39 \%$

Do research?

$20.40 \%$

Have a speciality?

$14.15 \%$
A) Language

I. How do you rate your knowledge of the English language?

Degree of competence $75.09 \%$

2. How did you learn the English Language? $\mathbf{7 5 . 4 7 \%}$ of respondents indicated more than one option

$\begin{array}{ll}\text { Self study } & 40.56 \% \\ \text { Diploma } & 70.75 \% \\ \text { Bachelor's } & 38.67 \%\end{array}$


Master's

Doctorate

In-service training

Experience

Intuition
$4.71 \%$

$0.94 \%$

$36.79 \%$

$55.66 \%$

$16.03 \%$

Other

Native speaker $\quad 4.71 \%$

Living abroad $\quad 4.71 \%$

Travelling $\quad 0.94 \%$

Courses $\quad 2.80 \%$

All primary, high school $\quad 1.80 \%$

3. How do you rate your knowledge of English speaking cultures?

Degree of competence $\mathbf{6 3 . 9 6 \%}$

4. How did you learn about English speaking cultures? $67.92 \%$ of respondents indicated more than one option

$\begin{array}{lr}\text { Self study } & 69.81 \% \\ \text { Diploma } & 29.24 \% \\ \text { Bachelor's } & 28.30 \% \\ \text { Master's } & 3.77 \% \\ \text { Doctorate } & 0.94 \% \\ \text { In-service training } & 20.75 \% \\ \text { Experience } & 50 \% \\ \text { Intuition } & 9.43 \%\end{array}$

Other

$\begin{array}{ll}\text { Travelling } & 4.71 \% \\ \text { Native Speaker } & 1.80 \% \\ \text { Living abroad } & 6.60 \% \\ \text { Books } & 0.94 \%\end{array}$

5. How do you rate your knowledge of how languages are learned?

Degree of competence: $72.64 \%$

6. How did you learn about language learning? $79.59 \%$ of respondents indicated more than one option 
Self study

Diploma

Bachelor's

Master's

Doctorate

In-service training

Experience

Intuition
$48.11 \%$

$62.26 \%$

$41.50 \%$

$8.49 \%$

$0.94 \%$

$49.05 \%$

$64.15 \%$

$21.69 \%$

Other

Education Certification secondary

$0.94 \%$

By creating programs for teacher training programs

$0.94 \%$

All primary, high school

$0.94 \%$

Cambridge

$0.94 \%$

B) DIAGNOSIS

7. Do you ever do any of the following in your job as an English Language teacher?

Evaluate students' language level

Evaluate students' learning achievements

Evaluate students' learning strategies and learning knowledge

Identify students' learning styles
$85.84 \%$

$76.41 \%$

$50 \%$

$60.37 \%$

8. How did you learn to do these activities? $77.35 \%$ of respondents indicated more than one option

Self study

Diploma

Bachelor's

Master's

Doctorate

In-service training

Experience

Intuition
$42.45 \%$

$56.60 \%$

$32.07 \%$

$9.43 \%$

$0.94 \%$

$50 \%$

$68.86 \%$

$27.35 \%$ 
Other
COTE
$1.80 \%$
Teacher's training course
$3.77 \%$
Diplomado IPN
$0.94 \%$

9. Who decides how and when you should do these activities? $40.56 \%$ of respondents indicated more than one option

You

Your head of department/area

Your work organization

C) Prescription

10. Do you ever do any of the following in your job as an English Language teacher? :

Help students to learn learning strategies

Choose learning materials and activities

Plan learning programmes and write objectives
$71.69 \%$

$43.39 \%$

$27.35 \%$

II. How did you learn to do these activities? $83.01 \%$ of respondents indicated more than one option

Self study

Diploma

Bachelor's

Master's

Doctorate

In-service training

Experience

Intuition
$51.88 \%$

$58.49 \%$

$35.84 \%$

II.32\%

$0.94 \%$

$47.16 \%$

$67.92 \%$

$22.64 \%$

Other

Diplomado IPN

$0.94 \%$

Courses, conferences or seminars

$3.77 \%$

12. Who decides how and when you should do these activities?
You
$83.01 \%$ 
Your head of department/area $\quad 36.79 \%$

Your institution

Other

Teacher's academy $\quad 0.94 \%$

Students

D) ReferRal to SpeCIALISTS ( 19.8 I\% OF RESPONDENTS DIDN'T ANSWER tHIS PART)

13. Do you ever do any of the following in your job as an English Language teacher? :

Help students to choose teachers suited to their learning styles

Help students to choose environments suited to their learning styles

Help students to choose activities suited to their learning styles

$67.92 \%$

14. How did you learn to do these activities? $62.26 \%$ of respondents indicated more than one option

Self study

Diploma

Bachelor's

Master's

Doctorate

In-service training

Experience

Intuition
$42.45 \%$

$39.62 \%$

$30.18 \%$

$6.60 \%$

$1.80 \%$

$30.18 \%$

$56.60 \%$

$16.98 \%$

Other

Diplomado IPN $\quad 0.94 \%$

Seminars

$3.77 \%$

15. Who decides how and when you should do these activities?

You

Your head of department/area

Your work organization
$69.81 \%$

$17.92 \%$

$16.03 \%$ 
E) AdMinISTRATION (I 7.92\% OF RESPONDENTS DIDN'T ANSWER THIS PART)

16. Do you ever do any of the following in your job as an English Language teacher? :

Keep records of students' language level evaluation

$73.58 \%$

Keep records of students' learning achievements

$45.28 \%$

Keep records of students' learning strategies and learning knowledge

$23.58 \%$

Keep records of students' learning styles

$19.81 \%$

Keep records of referrals to other teachers, environments or activities

$13.20 \%$

Advise students in writing of any of the above

$27.35 \%$

17. How did you learn to do these activities? $58.49 \%$ of respondents indicated more than one option

Self study

Diploma

Bachelor's

Master's

Doctorate

In-service training

Experience

Intuition
$37.73 \%$

$33.01 \%$

$28.30 \%$

$7.54 \%$

$0.94 \%$

$30.18 \%$

$59.43 \%$

$14.15 \%$

Other

Diplomado IPN $\quad 0.94 \%$

Courses, conferences $\quad 0.94 \%$

Part of job

$0.94 \%$

18. Who decides how and when you should do these activities? 28.30\% of respondents indicated more than one option

You

Your head of department/area

Your work organization

Students
$66.98 \%$

$24.52 \%$

$18.86 \%$

$0.94 \%$ 
F) General Practice

19. How would you classify your methodological approach to ELT?

Grammar- based (focus on the basic structure of language, such as grammar, vocabulary and pronunciation)

$53.77 \%$

Competency- based or functional context (focus on application of specific base language skills in areas needed to function in everyday life, at work, or in an academic context) $66.03 \%$

Whole language (integrated approach using listening, speaking, reading and writing in thematic contexts often introduced through learner generated content)

Participatory (focus on developing language and literacy skills to facilitate personal empowerment) $\quad 33.96 \%$ Content- based (focus on developing language to support learner success in specific content areas, such as vocational training) $\quad 26.41 \%$ Task.- based with a strong implicit grammar component

A mixure of all $1.80 \%$ Process - learning approach $\quad 0.94 \%$

20. How did you learn these approach(es)? $75.47 \%$ of respondents indicated more than one option

Self study

Diploma

Bachelor's

Master's

Doctorate

In-service training

Experience

Intuition
$43.93 \%$

$53.77 \%$

$34.90 \%$

$8.49 \%$

$0.94 \%$

$40.56 \%$

$63.20 \%$

$18.86 \%$ 
Other
Diplomado IPN
$0.94 \%$
School organization
$0.94 \%$

21 . Who decides which approach(es) to use? $32.07 \%$ of respondents indicated more than one option
You
$75.47 \%$
Your head of department/area
$32.07 \%$
Your work organization
$26.41 \%$
Coordinator
$0.94 \%$

22. How would you classify your personal approach to your work? Degree of agreement measured on a Likert scale I- 5 and expressed as a percentage of the accumulated score for complete agreement:

My job is enjoyable

$92.45 \%$

My job is challenging

$90.37 \%$

My job is helping people with the intellectual and emotional problems that arise from their learning My job is delivering a professional service of diagnosis, prescription, referral and record keeping.

$80.56 \%$

My main compensation for my job is the privilege

of knowing I have helped someone.

$60 \%$

My main compensation for my job is the privilege

of having status in the community

$54.33 \%$

My main compensation for my job is the salary I receive

$39.81 \%$

I am willing to accept my legal liability for the privilege of exercising my skill and judgment

$62.26 \%$

G) Professional Development

23. How did you get your job as an EFL teacher?

Internal appointment based on experience and references

$33.01 \%$ Internal appointment based on qualifications $59.43 \%$ Open competition based on experience and references $17.92 \%$ Open competition based on qualifications 
Contacts

$0.94 \%$

24. Who made the decision about your appointment? (indicate the authority p)

An academic

$66.03 \%$

An academic committee

$28.30 \%$

Other

Coordinator

$3.77 \%$

The academic head

$0.94 \%$

25. How do you keep up to date with new ideas?

Reading books on elt

$70.75 \%$

Reading journals on elt

$35.84 \%$

Going on courses

$85.84 \%$

Going to conferences

$79.59 \%$

Other

In-house training and development

$0.94 \%$

Seminars

$1.80 \%$

Internet

$1.80 \%$

Magazines

$0.94 \%$

26. How do you evaluate new ideas?

$\begin{array}{ll}\text { Try them out } & 91.50 \% \\ \text { Discuss them with colleagues } & 64.15 \% \\ \text { Read more about them } & 60.37 \%\end{array}$

Other

Discuss them with my students $\quad 1.80 \%$

27. Who evaluates your work as an EFL teacher?

You

Your head of department/area

An academic committee

Your work organization

Students

SS
$55.66 \%$

$51.88 \%$

$20.75 \%$

$25.47 \%$

$22.64 \%$

$1.80 \%$

28. How are you evaluated? 
Students' opinions

Students' results

Teaching load- Number of groups

Teaching load- Number of students

Administrative load

Research and publication

Keeping up to date

Participation in institutional committees

Contribution to society generally
$88.67 \%$

$61.32 \%$

$14.15 \%$

$13.20 \%$

$16.03 \%$

$10.37 \%$

$45.28 \%$

$13.20 \%$

$13.20 \%$ Other

By six month job review

$0.94 \%$

Myself

$0.94 \%$

28. What do you think of your work as an EFL teacher and your status in your institution?

'I enjoy my work. I have a challenging and interesting position at a good university and I really can not complain. I receive all the support I expect from the university.' (2)

'I think an EFL teacher should study and do research all the time. I am very happy with my status at the institution.' (3)

'I believe my work at the British Council over the last six years has given me the authority to make decisions regarding my career and my development in a principled way. I have grown quite enormously from my early years as a language teacher, and the fact that I am now pursuing an MA programme at a distance, and that the BC Mexico is helping me finance it, tells me that I have reached a comfortable position at my work. However, this is a competitive environment and I do not want to fall behind, so I hope I can develop further in the near future.' (5)

'I feel that the work of EFL teachers needs to be more recognized. I feel that in many ways my institution recognizes the work I do and helps promote the ELT profession in Mexico as a whole. However, there is always room for improvement.' (6)

'I consider that my work as an EFL teacher to be a really challenging and rewarding activity. Status: respected.' (7)

'I enjoy being an EFL teacher. I think that I do a good job. I also believe that 
within my institution my work is recognized as good.' (8)

'I am very privileged- I enjoy what I do a lot.' (9)

'I am glad of it because I am doing what I have been studying in the last 8

years and now I have a good status for it.' (I0)

'I am looking for new forms of improvement. However, my institution does not support me.' (II)

'I enjoy my work- I'm a humble teacher.' (I2)

'I really love my job. I mean to help people to achieve goals by using the English language. I am a professor.' (I3)

'I am happy with my job. I feel I have learned quite a lot in the past years and I am ready to share this with other persons ; other teachers and students.' (I4)

'I am a good teacher and people know it.' (I5)

'My status is good enough to arrange new projects for my department. I like my job and I know I can continue learning.' (I6)

'I think my work is very important. I feel heard and supported by my institution.' (19)

'I feel I have achieved my goal when I see students have learned. Teaching is a constant challenge but I feel really happy during class and preparing it.' (2I)

'I really like my job and I think that helps me to do it efficiently, besides the environment in the institution is great. I am pleased with my status as well.' (22)

'As a beginner in the area I think I do my work right I know I can teach cause I was a student not too long ago so I know what works and what does not about learning, though I know I have to take some courses to learn how to teach and I will. I am a good teacher but I know I can do it better.' (25)

'I think that working as an EFL teacher is a privilege because we are sharing our knowledge.' (26)

'I do love my job. I enjoy what I do moreover I do my best every single class However I would like to have more classes here. Furthermore I would be glad if you take into account what good and hard working teachers do.' (27) 
'I think my job is very rewarding. About my status, I think its ok.' (28)

'I think that it is necessary to change a lot the paradigms of many people who are part of the university to improve our teaching and learning process.' (29)

'I like teaching English because it is a way to help people.' (30)

'I do my best.' (3I)

'I think all teachers need constant training and I strongly believe this is my case. On the other hand, I am sure I am able to do this job and fulfill everything that's expected from me

Talking about my status well, I am not sure of what it is.' (32)

'It's OK, but it would be better if in our working organization they provide us with more ELT training.' (33)

'I think it is good' (36)

"I think it is a very rewarding work Not only do you receive "good" salary, but also the opportunity to share your knowledge, helping people to achieve what they need or want and above all, we as teachers can develop and keep up dated. Besides for those who were born to teach teaching is a way to live happily.' (37)

'It is highly rewarding and has become more challenging due to curriculum demands. My status right now is the result of more than 10 years of work and commitment.' (39)

'I am satisfied by and with my work.' (40)

'I really like teaching because I like helping people learn, and I like learning from them as well, and I know that's a challenge because I have to be up to date. Most of the time I think I'm not only a teacher of English but a teacher of everything.' (4I)

'I think my work gives results and I notice by the development of the students.' (42)

'I guess I'm doing it OK; every time trying to be better. I go abroad when I have the opportunity.' (43)

'Enjoyable but it is not well recognized by our university.' (44) 
'I love it.' (45)

'It's a completely enjoyable activity'. (46)

'I really think I can improve my ELT techniques.' (47)

'I find my job rewarding.' (48)

'I like my job, l've learned a lot, I've become more confident standing in front of and interacting with people. l'd like to feel more confident about my job.' (49)

'I think the ELT department in my university, and indeed also at the other universities where I have worked in Mexico (4 in total) has a low status in comparison with other departments. Their focus is on teachers having expert knowledge in their fields of study rather than being expert educators and this leads to ELT departments not receiving 'academic' recognition. At the same time, I think it is a situation which is in part self- generated. I have worked alongside native speakers who have been hired as teachers with no training whatsoever. Furthermore, as it is very easy to become a 'certified English teacher' (eg. CELTA, a one month course) this leads to a lot of amateurs rather than professionals in ELT jobs.' (50)

'I love the work I do. I think my institution knows I am a good teacher but never says or does anything to praise my work.' (5I)

'I really like my job as well as the opportunity to teach the language to others who need it. There are some things that can be improved about teaching. However, that's part of experience. Many things can be learnt on the way to work.' (53)

'Satisfactory in general. Frustrating because of the amount to teach in a very limited time. Status: I think I am considered a good dedicated teacher- sometimes too demanding??' (54)

'It is something enjoyable and I do not have a high position. I'm only a teacher, and a happy one.' (55)

'I think my work is always developing some new strategies in class as well as changing techniques according to the students' necessities. In my opinion, my status is as a known and professional teacher who worries and helps the students in and outside class.' (56)

'My work is highly regarded and challenging, I cherish it. The only drawback is the salary. I enjoy having certain teacher's privileges and the respect of my 
students although not all areas of society give teachers the respect we deserve.' (57)

'I'm very pleased with my work.' (58)

'Both aspects are very good.' (59)

'I really enjoy teaching but I don't think it gives a 'high status'. (60)

'It's a good job which gives you a lot of satisfaction.' (6I)

'I enjoy it; keeps me on my toes; it's in a public education institution serving the community.' (62)

'It's a challenging job or mission, but l'm not interested in my salary.' (65)

'I think it's the best job I could have gotten since I enjoy it a lot, and the rewards I get couldn't be found anywhere else.' (68)

'I love teaching English language, and I am happy to teach it at the IPN Cenlex. It's a privilege.' (69)

'I’m simply TOPS!' (70)

'I see my work as a small piece of achievement which fits into the goals set forth by my institution, and I am respected and taken into account for school projects, so my status is just as high as I want it to be.' (7I)

'I enjoy doing my work although it requires a lot of patience and innovation. It's tough when the material provided by the school is not enough or lacks quality. I've learned a lot by taking courses and reading, and this has helped me to face classroom problems.' (72)

'I think that we don't work in the other part that our work includes. We only teach.' (73)

'It is motivating because you work with and for people. But I don't think that my status as a teacher is recognized by the authorities.' (74)

'I think it is good, but needs to be improved.' (75)

'I like my job although sometimes we are not motivated in things like salary, benefits etc.'(76)

'I do my best every time I stand in front of my students, and I try not only 
being a good colleague but also a person on whom they are able to rely any time they need me.' (77)

'I think l've got a great subject to teach. I really enjoy it and feel well enough when the outcome is outstanding. As for my status, we are many teachers and we are entirely responsible for our group. We're treated with respect although I'm not completely satisfied with the wage.' (78)

'Nowadays I don't think teachers have the status we used to have 10 years ago, for instance. We are seen as part of the whole staff within the institution.' (80) 'It is not well paid.' (83)

'I like teaching but I don't care about the status.' (84)

'It's a really satisfying activity I enjoy working on and it's a pleasure to work for IPN.' (86)

'I'm trying to be professional in most of my job. Being prepared and researching are my principle goals as an English teacher. Planning and preparing lessons in sequences.' (87)

'Being an EFL teacher is something I really love doing and I'm good at it. My authorities trust me.' (88)

'I regard it as being enjoyable to work for an institution which gives the opportunity to plan my activities as I want without anything being imposed.' (89)

'Too much work, too little money' (90)

'If we are getting prepared all the time, why do the salaries stay so low?' (92)

'I've always tried to do my best. We're at the bottom of the ladder.' (93)

'It's very rewarding and I like it. As far as status is concerned, I'm disappointed because after studying at a university and having completed other courses I end up being 'The miss de ingles'.' (94)

'I love what I do. I feel appreciated by my institution.' (96)

'I consider I am a good EFL teacher. Nevertheless, I know there's still much to know and do to improve. I believe my colleagues and the school authorities think I am good at my work.' (97) 
'I think my work is great! I feel proud of it. As for my status, I think it is just OK.' (98)

'It is a very challenging activity. It is not as well rewarded as I would like it to be.' (99)

'I consider my work is well done although learners do not study as much as they should. This is the reason why they don't learn. I am a simple teacher working in my classroom with no other privileges.' (100)

'I think my activity as an English teacher contributes in some ways to students' knowledge growth. Concerning the status, I don't care. It seem like a not very serious research.' (IOI)

'I like my job and my principle compensation is knowing students learn and put into practice learning strategies.' (102)

'I like teaching but I think there's a lack of communication between teachers and authorities since we don't exchange in a clear way our opinions and state the objectives each one has.' (103)

'FANTASTIC!' ( I 04)

'We gave a great teaching load and our status in the institution is low.' (105)

'We aren't respected as the base of the institution and the management staff receives more benefits than teachers, which is totally unfair.' (I06) 